\title{
Angle-resolved electron energy loss spectroscopy in hexagonal boron nitride
}

\author{
Frédéric Fossard, ${ }^{1}$ Lorenzo Sponza, ${ }^{1}$ Léonard Schué, ${ }^{1,2}$ Claudio Attaccalite, ${ }^{3}$ François Ducastelle, ${ }^{1}$ \\ Julien Barjon, ${ }^{2}$ and Annick Loiseau ${ }^{1}$ \\ ${ }^{1}$ Laboratoire d'Etude des Microstructures, ONERA-CNRS, UMR104, Université Paris-Saclay, \\ Boîte Postale 72, 92322 Châtillon Cedex, France \\ ${ }^{2}$ Groupe d'Etude de la Matière Condensée, UVSQ-CNRS, UMR8635, Université Paris-Saclay, \\ 45 avenue des Etats-Unis, 78035 Versailles Cedex, France \\ ${ }^{3}$ Aix Marseille University and CNRS, CINAM, UMR7325, Campus de Luminy, case 913, 13288 Marseille, France
}

(Received 23 January 2017; revised manuscript received 28 July 2017; published 7 September 2017)

\begin{abstract}
Electron energy loss spectra were measured on hexagonal boron nitride single crystals employing an electron energy loss spectroscopic setup composed of an electron microscope equipped with a monochromator and an in-column filter. This setup provides high-quality energy-loss spectra and allows also for the imaging of energy-filtered diffraction patterns. These two acquisition modes provide complementary pieces of information, offering a global view of excitations in reciprocal space. As an example of the capabilities of the method we show how easily the core loss spectra at the $K$ edges of boron and nitrogen can be measured and imaged. Low losses associated with interband and/or plasmon excitations are also measured. This energy range allows us to illustrate that our method provides results whose quality is comparable to that obtained from nonresonant $x$-ray inelastic scattering but with advantageous specificities such as an enhanced sensitivity at low $\boldsymbol{q}$ and a much greater simplicity and versatility that make it well adapted to the study of two-dimensional materials and related heterostructures. Finally, by comparing theoretical calculations to our measures, we are able to relate the range of applicability of $a b$ initio calculations to the anisotropy of the sample and assess the level of approximation required for a proper simulation of our acquisition method.
\end{abstract}

DOI: 10.1103/PhysRevB.96.115304

\section{INTRODUCTION}

Two-dimensional (2D) materials are currently the object of many investigations concerning their electronic and optical properties. Most of the 2D materials, with the exception of graphene, are semiconductors with optical properties dominated by excitonic effects which depend on the number of layers and on the nature of the layer stacking [1]. In this landscape, $h$-BN displays a singular situation since it is a large-band-gap (about $6 \mathrm{eV}$ ) semiconductor with a honeycomb lattice similar to that of graphene in which boron and nitrogen alternate at the vertices of the honeycomb lattice. Optical measurements on $h$-BN are difficult because of the necessity to work in the far-UV range and require dedicated laser sources and detection devices [2-5]. Another possibility is to excite the system with electrons and to perform cathodoluminescence experiments [6-9]. Finally, photoemission excitation spectra can be obtained using VUV synchrotron radiation excitation [10]. All these experiments have clearly shown the importance of excitonic effects, in agreement with several theoretical studies [11-16], although their exact nature remains far from being fully clarified.

To go further in understanding excitonic properties, inelastic scattering techniques are useful and complementary tools to the above-cited optical spectroscopies. It is recalled here that the response to electronic excitations is characterized by the dynamical structure factor $S(\boldsymbol{q}, \omega)$, which is itself related to the dielectric response $\varepsilon(\boldsymbol{q}, \omega)$, where $\omega$ and $\boldsymbol{q}$ are, respectively, the energy (or frequency) and momentum variations during the involved scattering process $[17,18]$. As far as energy is concerned, optical techniques (absorption and photoluminescence) are very accurate but are confined to the $q \rightarrow 0$ limit. Recently, the full Brillouin zone (and beyond) of $h$-BN single crystals was explored by means of nonresonant inelastic x-ray scattering (NRIXS) experiments, and energy losses were recorded between a few and $40 \mathrm{eV}$. At low energy the resolution (down to $200 \mathrm{meV}$ ) made accessible the investigation of the near-edge excitonic regime for different values of $\boldsymbol{q}[19,20]$.

Such experiments can also be performed by using inelastic scattering of fast electrons [electron energy loss spectroscopy (EELS)]. EELS has suffered for a long time from low-energy resolution. That is no longer the case with the latest generations of electron microscopes, and this method can now be used to investigate not only the core-loss regime where energy variations are in the range $10^{2}-10^{3} \mathrm{eV}$ but also the low-loss regime, $\omega=1-50 \mathrm{eV}$. The current implantation of electron spectroscopy in transmission electron microscopes makes this technique particularly attractive as it opens the possibility for local investigations at the nanoscale, with no need for large samples, giving access to the impact of defects on the spectroscopic properties.

In this paper we present an EELS setup based on a transmission electron microscope (TEM) tweaked for angular-resolved electron spectroscopy and its application to a detailed study of $h$-BN single crystals. We show that the results are similar to those obtained using synchrotron x-ray sources (NRIXS) in terms of energy resolution, but this setup exhibits specific advantages: (i) It can be employed in two different acquisition modes, also allowing for the measurement of global maps of $S(\boldsymbol{q}, \omega)$ in the diffraction plane. (ii) It has privileged access to the small- $\boldsymbol{q}$ region of the Brillouin zone. (iii) The method is fast and can be applied to small samples, which opens the way to a broad field of applications, including 2D materials and their heterostructures. The methods are described in Sec. II. The results are presented in Sec. III, with those for the core-loss 
spectra at the boron $K$ edge in Sec. III A and those for the low-loss spectra in Sec. III B. Further documentation can be found in the Supplemental Material [21].

\section{TEM-EELS EXPERIMENTS}

In the past decade, the development of aberration-corrected TEM has brought new tools to the scientific community which are particularly suited to imaging thin materials [22,23]. Moreover, with the improvement of electron sources and monochromators associated with optimized spectrometers, EELS spectra can be recorded with atomic and sub-eV energy resolutions [24,25]. However, such systems still make compromises in order to increase the signal by integrating over a finite collecting solid angle [23] or by shining a very focused beam with a large illumination angle on the sample so that the angular-dependent information is averaged or truncated [26]. The techniques presented here avoid these disadvantages. They combine energy-filtered TEM (EFTEM) and EELS. The electron microscope is a Zeiss Libra 200 MC equipped with an electrostatic CEOS monochromator, an in-column $\Omega$ filter, and a Gatan ultrascan 1000 CCD camera. The microscope operates at $80 \mathrm{kV}$, and the monochromated beam gives a resolution of $100 \mathrm{meV}$ with the narrowest slit. The Köhler illumination ensures that the beam is parallel and that its convergence is kept below $80 \mu \mathrm{rad}$.

To measure the dynamical structure factor it is convenient to work within the diffraction plane of the microscope where the scattering angles can be related to the transferred momenta. Since the transferred momentum is much smaller than the momentum of the incoming beam, the relation between them is given by $q^{2} \simeq k^{2}\left(\theta^{2}+\theta_{E}^{2}\right)$, where $\boldsymbol{k}$ is the initial momentum, $\theta$ is the scattering angle, and $\theta_{E}$ is proportional to the energy loss [17]. For a given orientation of the sample, a data cube is built from the values of $\omega, q_{x}$, and $q_{y}$, with the incident beam being along the $z$ direction [27]. The component along this direction, $q_{E}=k \theta_{E}$, is negligible in general, except when $q$ is close to zero. This is illustrated in Fig. 1.

Two strategies have been applied to record this information and are described below. The first method consists of recording scattering patterns at given energy losses and stacking them in order to build the horizontal slices of the data cube. The main advantage of this procedure is to obtain the $q_{x}$ and $q_{y}$ values of the transferred momentum with the same resolution. The spectral resolution of the EFTEM experiment is determined by the exit slit of the energy filter, which selects a bandwidth in the energy-selecting plane [28]. The filtered electrons within this bandwidth form the scattering pattern. The intensity of the signal is an order of magnitude lower than the intensity of a usual diffraction pattern. As a consequence, the integrating time to record one slice is usually larger than $10 \mathrm{~s}$. Data are measured every $0.25 \mathrm{eV}$ to get a smooth spectrum which is used to subtract a power-law background in the $\omega$ direction of the data cube for every $\left(q_{x}, q_{y}\right)$ pixel.

In order to take full advantage of the monochromator and to obtain more quantitative results on the double $\omega-\boldsymbol{q}$ dependence, the second method is to combine the imaging capabilities of the projective system with the energy dispersion generated by the $\Omega$ energy filter in the microscope. A rectangular slit can be placed at the entrance of the filter
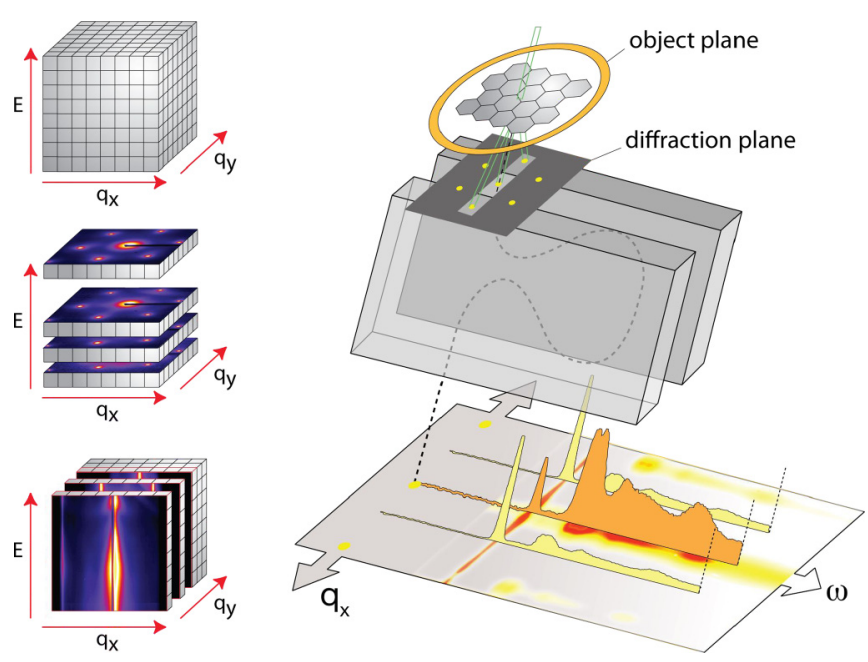

FIG. 1. Left: data cube in reciprocal space (top), $\left(E, q_{x}, q_{y}\right)$ data cube construction in EFTEM (middle), and $\left(E, q_{x}, q_{y}\right)$ data cube construction in the $\omega-\boldsymbol{q}$ map (bottom). Right: schematic principle of the $\omega-\boldsymbol{q}$ map acquisition.

in order to select a direction in reciprocal space $[29,30]$. The orientation of the slit is fixed, and its larger dimension is perpendicular to the direction of the energy dispersion. The orientation of the sample must be adjusted in order to align the slit with a specific crystallographic direction. This can be done using a tilt/rotation holder ensuring a $360^{\circ}$ rotation around the optical axis. Furthermore, since the slit is placed after the first projective system of the microscope, we can also rotate the diffraction pattern by changing the camera length.

The multipoles of the filter must be adjusted in order to keep the $q_{x}$ information in the direction of the slit while dispersing the energy of the electrons. The image recorded on the camera is therefore in the $\left(q_{x}, \omega\right)$ plane, as illustrated in Fig. 1 (right). Thus, by shifting the scattering pattern with the first projector system, we can scan the $q_{y}$ direction using discrete steps. In other words, the data cube is now built with vertical slices, as illustrated in Fig. 1 (bottom left).

It is worth noting that the $\boldsymbol{q}$ resolution in both experiments depends on several instrumental parameters as well as on sample specifications. Indeed, both experiments are diffraction experiments, and the broadening of the signal is related to the illumination angle, the camera length used, the optical design of the column, the width of the slits, and apertures [30]. One should also take into account the crystallinity of the sample over the diffracting area. In our case, the area is delimited by an aperture which gives a virtual circular area with a diameter of $70 \mathrm{~nm}$. Section III shows that the resolution along $q_{x}$ can be estimated to be about a few $10^{-2} \AA^{-1}$, whereas the thickness of the slice along $q_{y}$ in the $\omega-\boldsymbol{q}$ mode is about $0.2 \AA^{-1}$.

The procedures described above require $h$-BN samples with well-defined orientations. Three slabs have been cut by a focused ion beam from a $h$-BN single crystal [31] along

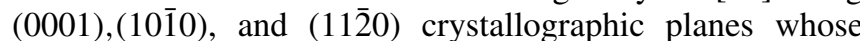
normal directions in the first Brillouin zone are $\Gamma A, \Gamma M$, and $\Gamma K$ (see Fig. 2). Further details are given in the Supplemental Material [21]. 

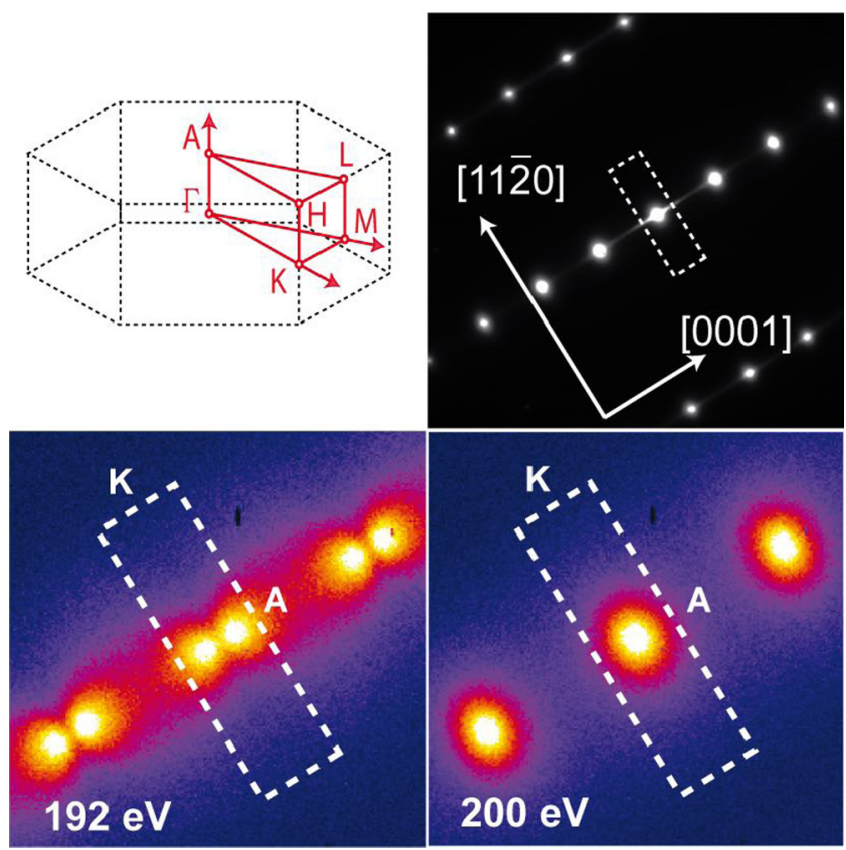

FIG. 2. Top: Hexagonal Brillouin zone and diffraction pattern of $h$-BN in a plane containing the hexagonal axis (direction $\Gamma A$ ) and the $\Gamma K$ direction. Bottom: energy-filtered scattering patterns recorded at $192 \mathrm{eV}$ (left) and at $200 \mathrm{eV}$ (right).

\section{RESULTS}

\section{A. Core losses at the boron $K$ edge}

Studying electron energy loss (EEL) at the boron $K$ edge is a textbook case for illustrating the potentialities of our technique. The different peaks are sharp and related to well-known transitions with typical symmetries between the deep $1 s$ level and the first unoccupied $\pi^{*}$ states $(192 \mathrm{eV})$ and $\sigma^{*}$ states (199 eV) [32-39]. Energy-filtered scattering patterns have been recorded in the 185-215 eV range for the three samples. Figure 2 presents the elastic diffraction pattern of the second sample with labeled directions as well as inelastic filtered patterns at the $1 s \rightarrow \pi^{*}$ energy $(192 \mathrm{eV})$ and the one close to the $1 s \rightarrow \sigma^{*}$ energy $(200 \mathrm{eV})$ obtained by EFTEM. Notice that in both cases diffuse intensities also occur around the Bragg peaks. This is due to double-scattering processes involving inelastic scattering and elastic Bragg scattering. At $192 \mathrm{eV}$, all diffraction spots are split into two symmetric lobes with the specific [0001] orientation (along the axis of the hexagonal cell). The corresponding $\omega-\boldsymbol{q}$ plot in the $\Gamma A$ direction is shown in Fig. 3. We have a clear illustration here of the anisotropy of the losses in the $1 s \rightarrow \pi^{*}$ transition, which can simply be explained as follows [32,38].

In the simplest single-electron picture the dynamic structure factor is given by

$$
S(\boldsymbol{q}, \omega)=\sum_{f}\left|\left\langle f\left|e^{i \boldsymbol{q} \cdot \boldsymbol{r}}\right| i\right\rangle\right|^{2} \delta\left(E_{f}-E_{i}-\hbar \omega\right),
$$

where $i$ and $f$ denote the one-electron initial and final states, respectively, with the differential cross section $d^{2} \sigma / d \Omega d E$ for electron scattering being equal to $4 S(\boldsymbol{q}, \omega) /\left(a_{0}^{2} q^{4}\right)$, where $a_{0}$ is the Bohr radius. The matrix element $\left\langle f\left|e^{i \boldsymbol{q} \cdot \boldsymbol{r}}\right| i\right\rangle$ reduces here to

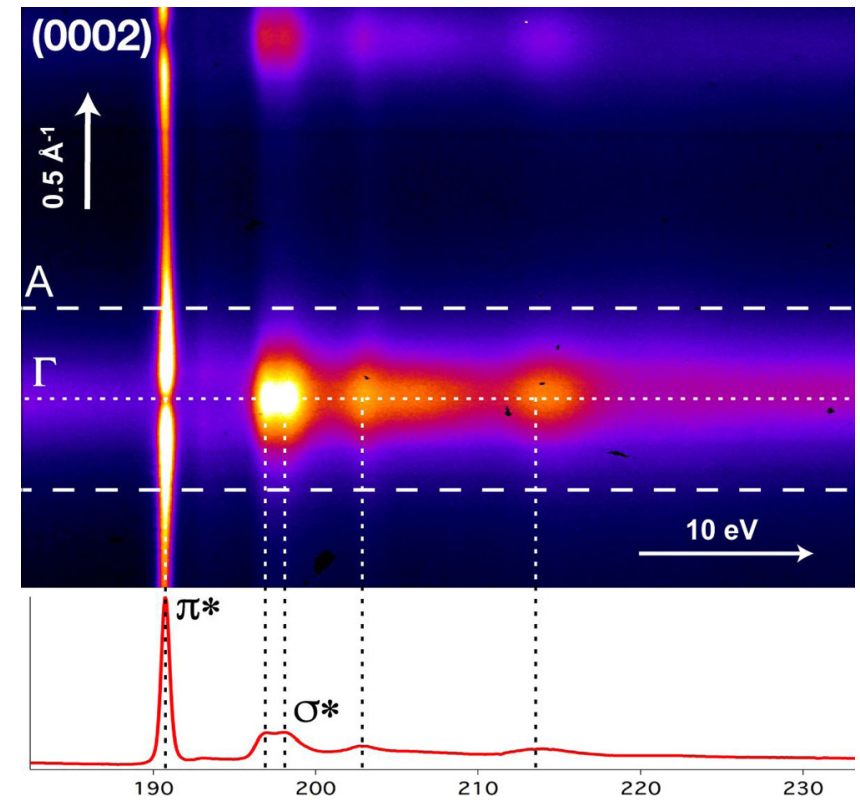

FIG. 3. Top: $\omega-\boldsymbol{q}$ image recorded in the $\Gamma A$ direction close to the boron $K$-edge energy. Dashed lines delimit the Brillouin zone. Bottom: related EELS spectrum integrated over the whole Brillouin zone. Dotted lines indicate the Brillouin zone section and significant edge structures.

the matrix element between the core boron $1 s$ function and the conduction-band states. In the case of $h$-BN the conduction states at low energy are concentrated on the boron atoms, more precisely on their $\pi_{\|}$states pointing along the hexagonal axis [16], so that, finally, within the dipolar approximation, we have to calculate the dipolar matrix element $\left\langle\pi_{\|}|\boldsymbol{q} \cdot \boldsymbol{r}| 1 s\right\rangle$. Because of the symmetry of the $\pi_{\|}$state, only the component of $\boldsymbol{r}$ along the hexagonal axis survives, so that we expect that $S(\boldsymbol{q}, \omega) / q^{4} \simeq q_{\|}^{2} / q^{4}=\cos ^{2} \alpha / q^{2}$, where here $\alpha$ is the angle between $\boldsymbol{q}$ and the hexagonal axis [32]. This means that the symmetry of the scattered intensity around the origin should be similar to that of the $\pi$ electron density itself. This is clearly the case, as shown in Fig. 2 and in Fig. 4, where an enlargement of the central lobes is shown.

More precisely, let us decompose the scattering wave vector $\boldsymbol{q}$ into its component in the diffraction plane $\overline{\boldsymbol{q}}$ and its inelastic component $\boldsymbol{q}_{E}$ along the incident beam, normal to this plane [30]. Then the scattering cross section measured in the diffraction plane is proportional to $\overline{\boldsymbol{q}}^{2} \cos ^{2} \alpha /\left(\overline{\boldsymbol{q}}^{2}+q_{E}^{2}\right)^{2}$. This induces an intensity dip along the hexagonal lattice, with a width equal to $2 q_{E}$, when approaching the origin. Actually, in this limit $\overline{\boldsymbol{q}} \rightarrow 0$, the scattering vector is normal to the diffraction plane and therefore in the nodal plane of the $\pi$ orbital. We can calculate $q_{E}$, which is equal to $E / \hbar v_{0}$, where $E$ is the energy loss and $v_{0}$ is the electron velocity determined by the accelerating tension of the microscope. In our case, $q_{E} \simeq 0.20 \AA^{-1}$, and it can be seen in Fig. 4 that the above formula fits the measured profiles perfectly. This indicates that the (angular) resolution in $q$ space is very good. It is estimated to a few $10^{-2} \AA^{-1}$.

At higher energy $(200 \mathrm{eV})$ the diffraction pattern is modified, with an intensity much more isotropic and with an 

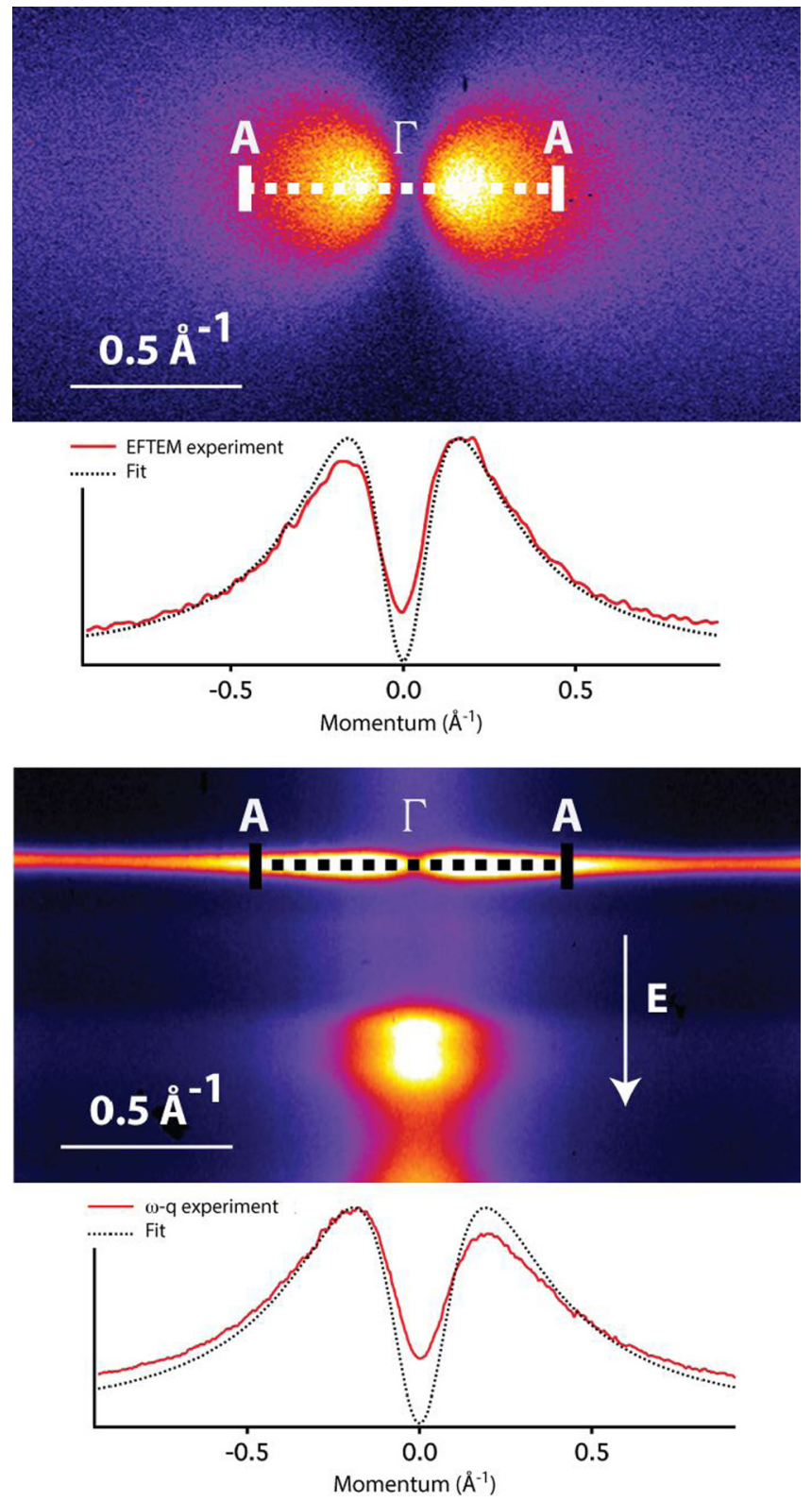

FIG. 4. Enlargement of the inelastic scattered intensity corresponding to the $\sigma \rightarrow \pi^{*}$ transition and related profile in the $\Gamma A$ direction in EFTEM at $192 \mathrm{eV}$ (top) and the $\omega-\boldsymbol{q}$ plot (bottom). The fit is made using a profile function proportional to $\overline{\boldsymbol{q}}^{2} /\left(\overline{\boldsymbol{q}}^{2}+q_{E}^{2}\right)^{2}$, with $q_{E}=0.20 \AA^{-1}$.

extension in the basal plane typical of the appearance of $\sigma$ states, as also discussed by Leapman et al. [32].

The difference between the two regimes is even more obvious when looking at the $\omega-\boldsymbol{q}$ plot shown in Fig. 3. It can be noticed that the $\pi^{*}$ peak at low energy is separated from a quasicontinuum starting with the $\sigma^{*}$ peak at higher energy which is typical of excitonic behavior. The splitting of the main $\sigma^{*}$ peak apparent in the $\omega-\boldsymbol{q}$ plot probably also has an excitonic origin [40]. The corresponding EEL spectrum for $\overline{\boldsymbol{q}}$ along the hexagonal axis is shown in Fig. 4. Actually, the presence of a core hole is important here, and the single-electron description should be improved. For instance, the authors of reference [38] calculated the full dielectric constant using the
Bethe-Salpeter formalism [41] and found good agreement with the experimental data.

The $\omega-\boldsymbol{q}$ plots have also been obtained for energies close to the nitrogen $K$ edge (see the Supplemental Material [21]). The core hole is on the nitrogen atom, but the electron in the conduction band is still concentrated on the boron atoms. Excitonic effects and oscillator strengths are therefore expected to be weaker, which is the case: The measured EELS signals are much weaker and therefore less accurate, and the spectra show broader and less "atomiclike" features, in agreement with previous studies $[34,35,37,40,42]$.

\section{B. Low-loss region}

The low-loss regime is related to the loss function equal to $-\operatorname{Im}[1 / \varepsilon(\boldsymbol{q}, \omega)]$ and proportional to $S(\boldsymbol{q}, \omega) / q^{2}$, so that the differential scattering cross section is proportional to $-1 / q^{2} \operatorname{Im}[1 / \varepsilon(\boldsymbol{q}, \omega)]$. The peaks of the loss function are frequently associated with plasmons. Two energy ranges are generally distinguished, with a $\pi$ plasmon peak in the $6-8 \mathrm{eV}$ range and a $\sigma+\pi$ peak at about $25 \mathrm{eV}$ for bulk $h$-BN and also for graphite [43-47], the position and intensity of the latter peak being strongly dependent on the number of sheets in thin samples. The position of some structures can also be associated with specific interband transitions, particularly if they are correlated with the behavior of $\varepsilon(\boldsymbol{q}, \omega)$ itself through Kramers-Kronig analyses [43], but some controversy has appeared recently between these two interpretations concerning the nature of the observed signals in 2D systems such as graphene [48-51]. Actually, deriving well-defined dispersion relations and deciding between the two possibilities is not obvious. In most cases the excitations have a mixed character reinforced by the fact that local field and many-body effects are important, so that the discussion has a somewhat semantic character. Nevertheless, accurate calculations based on the Bethe-Salpeter equation are now available and recently were used successfully to analyze NRIXS experiments [19,20], notably in relation to specific excitonic peaks arising at $\boldsymbol{q}$ outside the first Brillouin zone.

The capabilities of our approach in the low-loss regime are well exemplified by Figs. 5 and 6, which show images produced by the two recording techniques of the spectroscopic setup. In Fig. 5 we present $\omega-\boldsymbol{q}$ maps (top panels) as well as the corresponding loss function along the $\Gamma A, \Gamma M$, and $\Gamma K$ directions in the range $0-25 \mathrm{eV}$ (bottom panels). Along $\Gamma K$ the low-energy peak moves upwards, from about 8 to about $12 \mathrm{eV}$, whereas along $\Gamma M$ this peak splits when $\boldsymbol{q}$ approaches the Brillouin zone boundary at $M$. The two spectra instead look similar at small $\boldsymbol{q}$ and coincide at $\boldsymbol{q} \approx 0$. Along $\Gamma A$ the peaks do not disperse significantly as a manifestation of the weak interplanar interaction. The $\Gamma A$ spectrum differs significantly from the other two because of the anisotropy of the dielectric function $\varepsilon_{\|}(\omega) \neq \varepsilon_{\perp}(\omega)$. As a complementary piece of information, we also have access to scattering patterns at fixed energy in both basal and prismatic orientations. As an instructive example, Fig. 6 shows two patterns taken by averaging the signal in the basal plane within $1 \mathrm{eV}$ around 8 and $12 \mathrm{eV}$. Although multiple-scattering effects spoil the signal outside the Brillouin zone [52], inside it the diffuse intensity, which is the relevant quantity here, is clearly detectable. These 

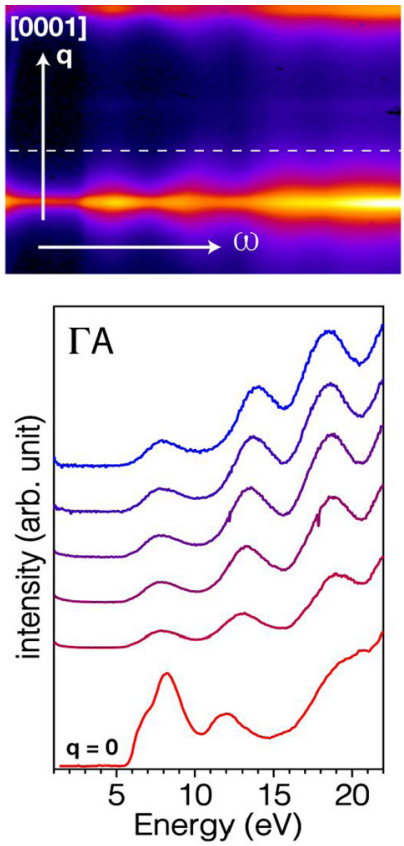
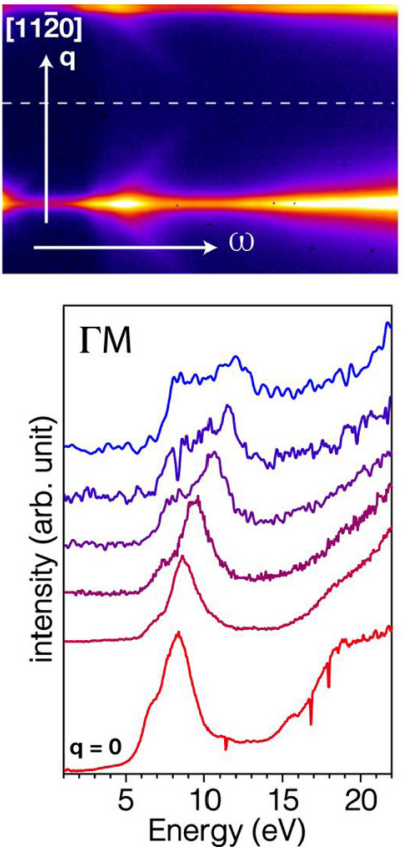
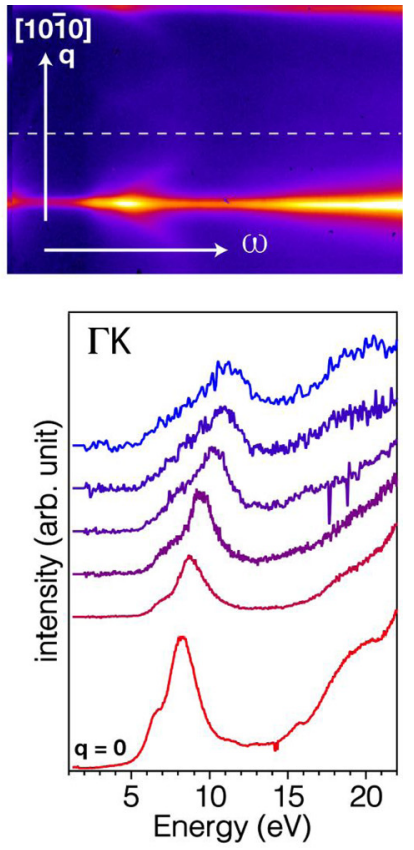

FIG. 5. Top: $\omega-\boldsymbol{q}$ plots as measured (dashed lines indicate the Brillouin zone boundaries). Bottom: loss function spectra along high-symmetry directions. Spectra at $q=0$ are shown separately, while the others are distributed every $20 \%$ of the Brillouin zone.

maps clearly show the differences in the dispersion of the diffuse intensity observed from the $\omega-\boldsymbol{q}$ maps along $\Gamma K$ and $\Gamma K$ at about 8 and $12 \mathrm{eV}$.

In the following, we illustrate the specific characteristics of our method applied to the low-loss regime by discussing its complementarity to x-ray spectroscopy [19]. Then we compare $a b$ initio calculations against our data with the intent to assess the level of approximation required for an accurate description.

\section{Comparing EELS and NRIXS}

The small- $q$ regime is particularly appealing for discussing the strong points of EELS with respect to NRIXS. It is recalled here that the scattering cross section is proportional to $q^{2} \operatorname{Im}[-1 / \varepsilon(\boldsymbol{q}, \omega)]$ in the case of NRIXS and proportional to $\left(1 / q^{2}\right) \operatorname{Im}[-1 / \varepsilon(\boldsymbol{q}, \omega)]$ in the case of EELS. This makes EELS particularly suited for probing small exchanged momenta. This complementarity is evident when comparing our data (Fig. 5) to NRIXS data [19]. In Fig. 5, the signal starts becoming
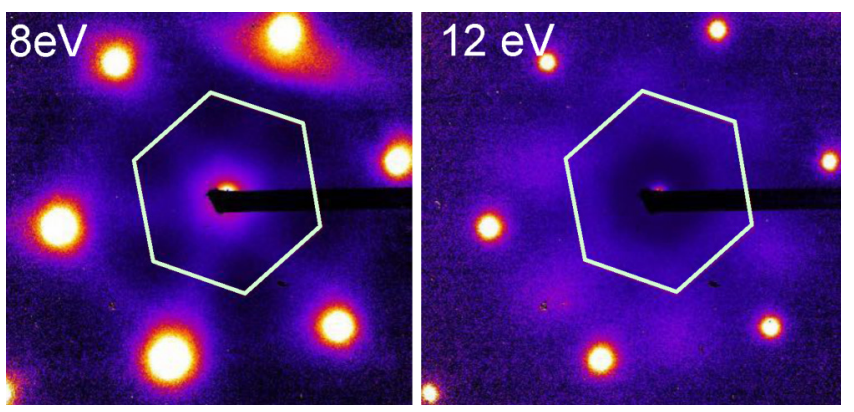

FIG. 6. Energy-filtered scattering patterns measured at different energies in the low-loss regime. The Brillouin zone boundary is marked in white. noisy at $q \approx 1.0 \AA^{-1}$ (around $60 \%$ of $\Gamma K$ and $80 \%$ of $\Gamma M$ ); conversely, in Fig. 1 of Ref. [19], the signal is extremely weak up to $0.6 \AA^{-1}$, which is even beyond the zone border along $\Gamma A$. Being intrinsically very sensitive in the small- $q$ regime, our EELS method can bridge the gap between optical measurements (very precise but limited to $q \rightarrow 0$ ) and the $\mathrm{x}$-ray experiments (sensitive at large $\boldsymbol{q}$ ). It is hence a powerful and versatile tool for accurately investigating the dielectric properties inside the Brillouin zone, notably in the vicinity of $\boldsymbol{q}=0$, where excitonic effects exhibit peculiar characteristics in 2D materials and thin films $[15,53]$.

However, in the optical limit results have to be analyzed carefully. When comparing the $\Gamma K$ and $\Gamma M$ directions at $\boldsymbol{q} \approx 0$, it is clear that the scattered intensity becomes isotropic in the basal plane (see bottom panels in Fig. 5), as expected from physical considerations and in agreement with theoretical calculations $[45,54,55]$. In the same limit, the spectrum along $\Gamma A$ differs from the in-plane ones because of the anisotropy of $\varepsilon(\boldsymbol{q}, \omega)$, but it exhibits an intense structure at $8 \mathrm{eV}$ which is unexpected. In fact, most calculations predict a much weaker intensity for structures below $12 \mathrm{eV}[45,54,55]$. In the core losses, the $\boldsymbol{q} \rightarrow 0$ limit was problematic because of the $q_{E}$ component. But here $q_{E}$ can be neglected as it is of the order of $0.005 \AA^{-1}$ owing to the lower energy loss. In this case, actually, the problem comes from the width $\Delta q_{y}$ of the slit used in the $\omega-\boldsymbol{q}$ mode, which is about $0.20 \AA^{-1}$. When collecting data along $\Gamma M$ and $\Gamma K$, the slit lies parallel to the basal plane, where $h$-BN is isotropic at $\boldsymbol{q} \rightarrow 0$. Instead, when measuring along the $\Gamma A$ direction, $q_{x}$ is parallel to $z$, while $q_{y}$ still lies parallel to the basal plane. This leads to a mixture of $\varepsilon_{\|}$and $\varepsilon_{\perp}$, the latter being predominant. This explains why the 8 $\mathrm{eV}$ structure in the $\boldsymbol{q} \approx 0 \Gamma A$ spectrum looks so similar to the equivalent peak in the basal-plane spectra and is instead washed out at higher $\boldsymbol{q}$. 

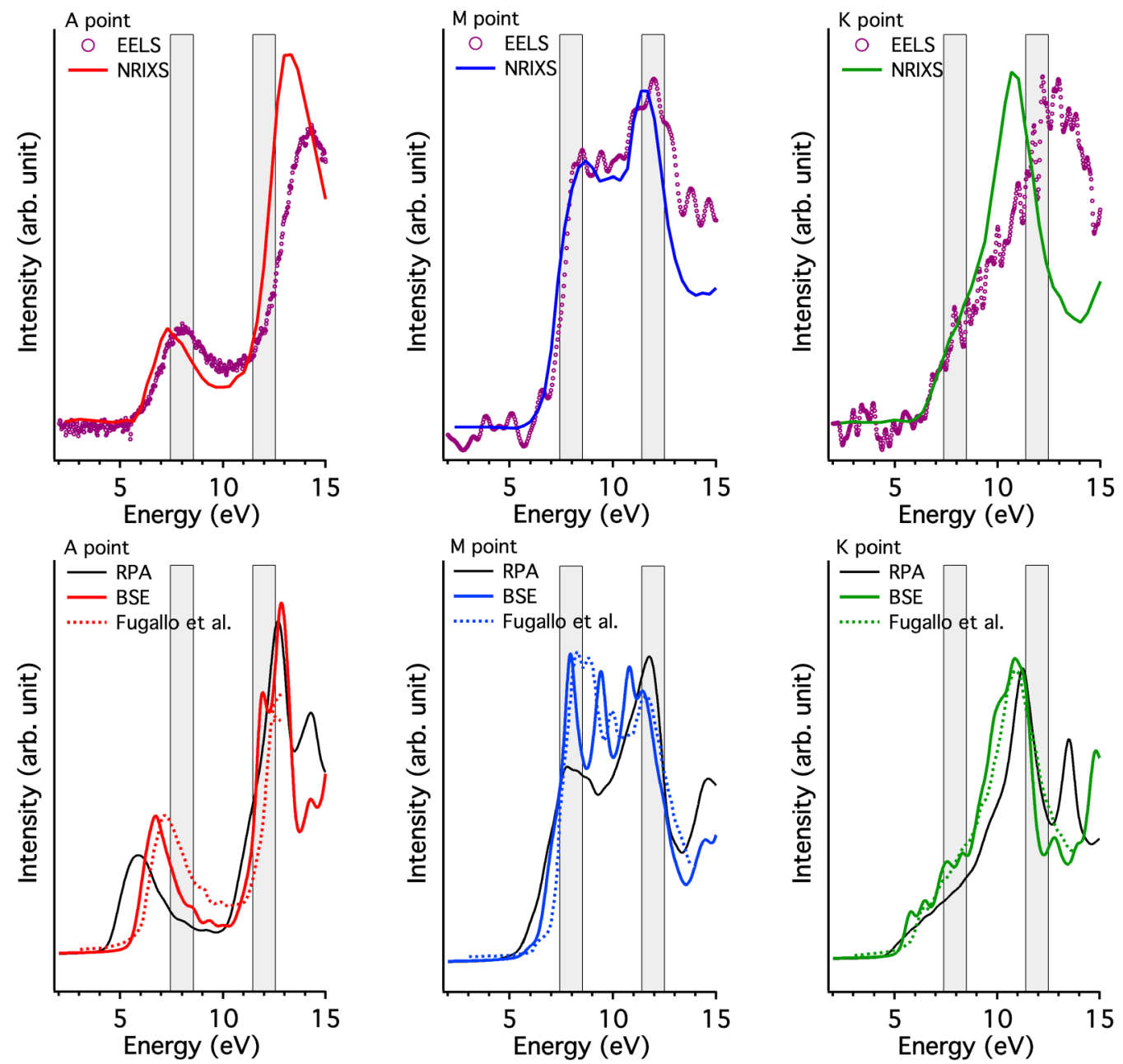

FIG. 7. Top: comparison between our EELS and NRIXS [19] measures. Bottom: comparison between theoretical curves (ours and Fugallo's [20]). Our theoretical spectra have been convoluted with a Gaussian with a width of $0.2 \mathrm{eV}$.

We now point out that the "low- $q$ " region is actually wide enough to explore the entire first Brillouin zone. In the top panels of Fig. 7 we report the comparison between our data and NRIXS data [19] at the high-symmetry points $A, M$, and $K$ located at the zone boundary. In these points both techniques have reasonably high accuracy. Gray shaded strips delimit the energy intervals for the averages done in obtaining the diffraction maps in Fig. 6. The good agreement between the two techniques demonstrates that EELS gives accurate results for $\boldsymbol{q}$ as large as the zone border. Together with the considerations above, this shows that EELS ensures high-quality data inside the whole Brillouin zone.

\section{Comparison with theoretical calculations}

As discussed by Galambosi et al. [19] and by Fugallo et al. [20], several theoretical ingredients are necessary to account for all the details of inelastic scattering experiments. This is especially true for excitonic features that can be correctly simulated only by going beyond the independentparticle approach [random-phase approximation (RPA)] and solving instead the Bethe-Salpeter equation (BSE). In the works cited above, the authors used BSE to investigate the origin of specific peaks, in particular for momenta outside the first Brillouin zone. However, BSE is computationally very demanding with respect to RPA. The question we want to answer in this section is then, Is the BSE accuracy indispensable for describing our EELS data?

All calculations were carried out with the code GPAW [56]. Structural parameters are $a=2.50 \AA$ and $c=6.5 \AA$, in agreement with those obtained from measured diffraction patterns. The ground-state density was obtained sampling the Brillouin zone with a $6 \times 6 \times 2 \Gamma$-centered $k$-point grid and including plane waves up to $900 \mathrm{eV}$. The Perdew-Burke-Ernzerhof approximation was adopted for the exchange-correlation potential [57]. The RPA loss function was computed in all $q$ points of a $24 \times 24 \times 8 \Gamma$-centered grid, including 20 bands and having a cutoff of $60 \mathrm{eV}$. The diffraction patterns at energy $E$ were obtained by first averaging the computed spectra in the range $E \pm 0.5 \mathrm{eV}$ and then interpolating the result on a sufficiently dense mesh (50 points). The BSE was solved for six valence bands and eight conduction bands on a $12 \times 12 \times 4(8)$ $\Gamma$-centered $q$-point grid for $\mathbf{q}$ in plane (out of plane). A scissor operator of $1.73 \mathrm{eV}$ (derived from the average $\mathrm{GW}$ correction across the gap) was applied to the Perdew-Burke-Ernzerhof energies. A cutoff of $60 \mathrm{eV}$ and 20 bands were included to converge the dielectric constant entering in the direct term of the excitonic Hamiltonian. 
In the bottom panel of Fig. 7 we report our RPA and BSE curves together with data extracted from the work by Fugallo and coworkers [20] used as a validation benchmark. The main differences between RPA and BSE spectra are (i) a redistribution of the spectral weight, notably in the first peaks at $\boldsymbol{q}=M$, and (ii) a shift of the low-energy peak at $\boldsymbol{q}=A$. These excitonic effects have already been discussed in the literature [19,20], and further details can be found in the Supplemental Material [21]. Indeed, to account for these relatively tiny effects BSE is unavoidable, but what we want to stress here is that at $\boldsymbol{q}=M$ and $\boldsymbol{q}=K$, not only are the main structures correctly reproduced already at the RPA level, as expected, but they also fall in the right position. Moreover, this is true in the whole basal plane (see Fig. 6 of the Supplemental Material [21]).

The physical reason is that on the basal plane the quasiparticle normalization due to $e-e$ scattering (here approximated by the scissor operator) is almost entirely canceled by the $e$-h attraction. From a practical point of view this means that, as long as $\boldsymbol{q}$ lies on the basal plane, the RPA is good enough to describe the dispersion of the loss function, and it can be used successfully to simulate both acquisition methods. Instead, along $\Gamma A$, the anisotropy of the electronic screening spoils this mutual cancellation, leading to a misalignment of the first peak. This is clearly shown in the left panels of Fig. 7, where the measured data exhibit a local maximum at $8 \mathrm{eV}$, whereas the RPA spectrum is almost vanishing. Both sets of data instead overlap pretty well at $\sim 12 \mathrm{eV}$. As a consequence, the loss function with $\boldsymbol{q} \| \Gamma A$ cannot be computed at the RPA level in a large energy range with the right alignment of all peaks. In particular
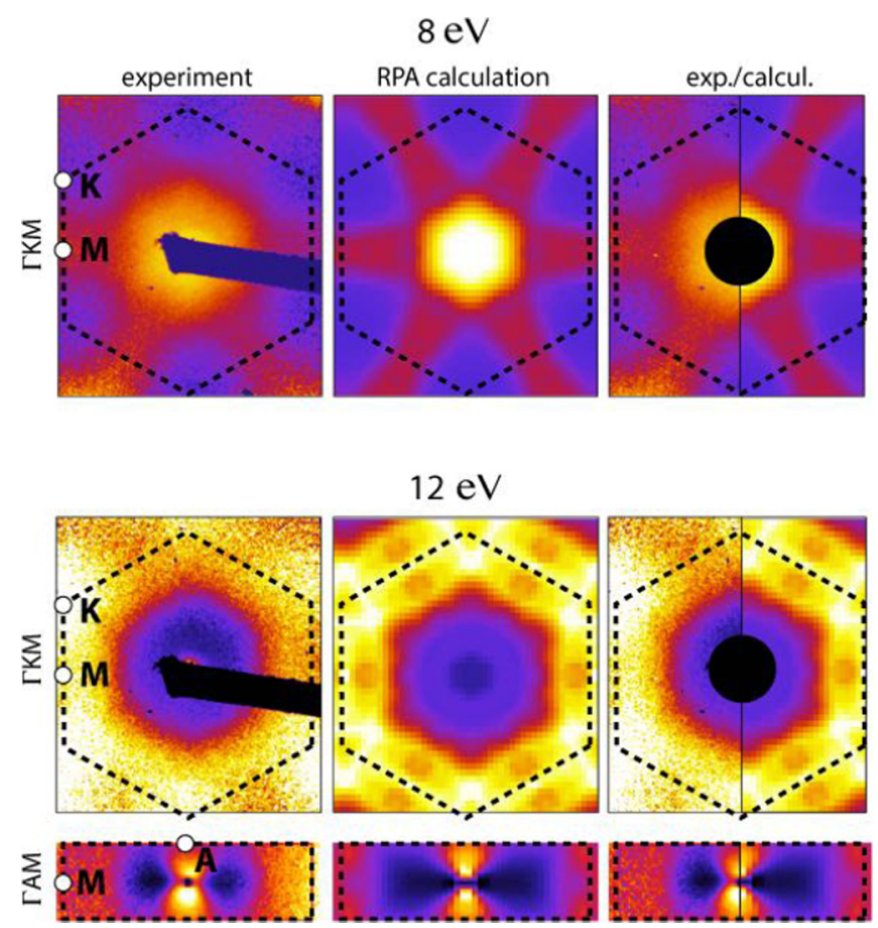

FIG. 8. Experimental and calculated loss functions (scattered intensities multiplied by $q^{2}$ ) at $8 \mathrm{eV}$ for the $\Gamma K M$ plane and at $12 \mathrm{eV}$ for the $\Gamma K M$ and $\Gamma A M$ planes. Dashed lines delimit the Brillouin zone. this is a problem when simulating diffraction patterns (e.g., in the $\Gamma A M$ plane) since a single plot includes perpendicular $\boldsymbol{q}$ (correctly aligned), parallel $\boldsymbol{q}$ (wrongly aligned), and all momenta in between. The right alignment in all directions can be surely ensured by BSE, but the heavy computational cost of the method hinders the applicability to the simulation of diffraction patterns. Moreover, the energy average carried out would wash out most of the weight redistribution, which makes the use of BSE quite disproportionate.

In order to display the quality of the RPA, in Fig. 8 we report simulated and measured energy-filtered dispersion patterns of the loss function at energies of 8 and $12 \mathrm{eV}$ in the basal plane (top and middle panels). At low energy $(8 \mathrm{eV})$, the patterns in the $\Gamma K M$ plane are characterized by an intensity concentrated at the origin, with diffuse arms pointing along the $\Gamma M$ directions, whereas at higher energy the intensity is higher close to the Brillouin zone boundary, with diffuse arms along the $\Gamma K$ directions. This is consistent with a simple analysis in terms of $\pi-\pi^{*}$ excitations in this energy regime. At low energy the transitions are mainly direct transitions $(\boldsymbol{q} \simeq 0)$, whereas they are indirect at higher energy [19]. In the bottom panel of Fig. 8, we show similar maps at $12 \mathrm{eV}$ in the $\Gamma A M$ plane, which contains therefore the $\Gamma A$ direction. This is possible because at this energy the signal is accidentally well aligned in all directions. As expected from the spectra in Fig. 5, the intensity at low $q$ is maximum in the $\Gamma A$ direction.

\section{CONCLUSION}

In summary we demonstrated that momentum-resolved EELS allows us to obtain accurate information on the electronic excitation spectra for core losses as well as for low losses. We illustrated this by treating the case of $h$-BN for which NRIXS data are available. The energy-filtered diffraction patterns provide a global view of anisotropy effects in $\boldsymbol{q}$ space, whereas the $\omega-\boldsymbol{q}$ plots allow us to map the symmetries of the losses as a function of the energy transferred to the material. The case of core losses related to the excitonic $\sigma-\pi^{*}$ transitions at the boron $K$ edge was shown to be particularly spectacular. In the case of low losses, our results confirm those of inelastic x-ray scattering experiments, allowing us to point out some advantages specific of our method.

EELS is an efficient technique complementary to other inelastic scattering tools such as NRIXS, despite their comparable energy resolution of about 100-200 meV. Indeed, their accuracy in $\boldsymbol{q}$ space is different. As EELS performs much better at low $q$, it opens the way to make contact with optical measurements. In particular, in 2D materials and heterostructures excitonic effects are important and present peculiar characteristics close to $\boldsymbol{q}=0[15,53]$; it is therefore highly desirable to have a tool adapted for measuring the dispersion of the excitonic levels in the low- $\boldsymbol{q}$ regime. Moreover, EELS within an electron microscope has the nonnegligible advantage of permitting fast and local experiments at the nanoscale.

Finally, with the support of $a b$ initio calculations at the RPA and BSE levels, we pointed out that in $h$-BN $e-e$ and $e-\mathrm{h}$ effects almost cancel out in excitations with $\boldsymbol{q}$ parallel to the layers, while the former dominates for $\boldsymbol{q} \| \Gamma A$. The practical consequence is that, as long as the exchanged momentum lies in plane, RPA calculations describe well energy loss spectra 
and diffraction patterns in a pretty large energy range. Instead, when excitations perpendicular to the planes are involved, one has to rely on BSE calculations to correctly align all the peaks. This is particularly problematic when simulating diffraction patterns because of the high computational cost of these calculations.

\section{ACKNOWLEDGMENTS}

T. Taniguchi and K. Watanabe from NIMS are warmly acknowledged for providing a reference HPHT crystal. The authors want to thank D. Troadec from IEMN for the FIB samples preparation and P. Wachsmuth, G. Benner, and U. Kaiser for very useful discussions on $\omega-\boldsymbol{q}$ maps. H. Amara is acknowledged for many fruitful discussions, G. Fugallo is acknowledged for the careful comparison with her theoretical results, and E. Gaufreès is thanked for a careful reading of the manuscript. The research leading to these results has received funding from the European Union H2020 Programme under Grant Agreement No. 696656 GrapheneCore1. We acknowledge funding from the French National Research Agency through Project No. ANR-14-CE08-0018.
[1] F. Xia, H. Wang, D. Xiao, M. Dubey, and A. Ramasubramaniam, Two-dimensional material nanophotonics, Nat. Photonics 8, 899 (2014).

[2] K. Watanabe, T. Taniguchi, and H. Kanda, Direct-bandgap properties and evidence for ultraviolet lasing of hexagonal boron nitride single crystal, Nat. Mater. 3, 404 (2004).

[3] P. Jaffrennou, J. Barjon, J.-S. Lauret, A. Loiseau, F. Ducastelle, and B. Attal-Tretout, Origin of the excitonic recombinations in hexagonal boron nitride by spatially resolved cathodoluminescence spectroscopy, J. Appl. Phys. 102, 116102 (2007).

[4] K. Watanabe and T. Taniguchi, Jahn-Teller effect on exciton states in hexagonal boron nitride single crystal, Phys. Rev. B 79, 193104 (2009).

[5] G. Cassabois, P. Valvin, and B. Gil, Hexagonal boron nitride is an indirect bandgap semiconductor, Nat. Phot. 10, 262 (2016)

[6] P. Jaffrennou, J. Barjon, T. Schmid, L. Museur, A. Kanaev, J.-S. Lauret, C. Y. Zhi, C. Tang, Y. Bando, D. Golberg, B. AttalTretout, F. Ducastelle, and A. Loiseau, Near-band-edge recombinations in multiwalled boron nitride nanotubes: Cathodoluminescence and photoluminescence spectroscopy measurements, Phys. Rev. B 77, 235422 (2008).

[7] A. Pierret, J. Loayza, B. Berini, A. Betz, B. Plaçais, F. Ducastelle, J. Barjon, and A Loiseau, Excitonic recombinations in $h$-BN: From bulk to exfoliated layers, Phys. Rev. B 89, 035414 (2014).

[8] R. Bourrellier, M. Amato, L. Henrique G. Tizei, C. Giorgetti, A. Gloter, M. I. Heggie, K. March, O. Stéphan, L. Reining, M. Kociak, and A. Zobelli, Nanometric Resolved Luminescence in $h$-BN Flakes: Excitons and Stacking Order, ACS Photonics 1, 857 (2014).

[9] L. Schue, B. Berini, A. C. Betz, B. Placais, F. Ducastelle, J. Barjon, and A. Loiseau, Dimensionality effects on the luminescence properties of hBN, Nanoscale 8, 6986 (2016).

[10] L. Museur, G. Brasse, A. Pierret, S. Maine, B. Attal-Tretout, F. Ducastelle, A. Loiseau, J. Barjon, K. Watanabe, T. Taniguchi, and A. Kanaev, Exciton optical transitions in a hexagonal boron nitride single crystal, Phys. Status Solidi RRL 5, 214 (2011).

[11] B. Arnaud, S. Lebègue, P. Rabiller, and M. Alouani, Huge Excitonic Effects in Layered Hexagonal Boron Nitride, Phys. Rev. Lett. 96, 026402 (2006).

[12] L. Wirtz, A. Marini, and A. Rubio, Excitons in Boron Nitride Nanotubes: Dimensionality Effects, Phys. Rev. Lett. 96, 126104 (2006).
[13] L. Wirtz, A. Marini, M. Grüning, C. Attaccalite, G. Kresse, and A. Rubio, Comment on "Huge Excitonic Effects in Layered Hexagonal Boron Nitride", Phys. Rev. Lett. 100, 189701 (2008).

[14] B. Arnaud, S. Lebègue, P. Rabiller, and M. Alouani, Arnaud, Lebègue, Rabiller, and Alouani Reply, Phys. Rev. Lett. 100, 189702 (2008).

[15] P. Cudazzo, L. Sponza, C. Giorgetti, L. Reining, F. Sottile, and M. Gatti, Exciton Band Structure in Two-Dimensional Materials, Phys. Rev. Lett. 116, 066803 (2016).

[16] T. Galvani, F. Paleari, H. P. C. Miranda, A. Molina-Sánchez, L. Wirtz, S. Latil, H. Amara, and F. Ducastelle, Excitons in boron nitride single layer, Phys. Rev. B 94, 125303 (2016).

[17] R. F. Egerton, Electron Energy-Loss Spectroscopy in the Electron Microscope, 3rd ed. (Springer, New York, 2011).

[18] K. Sturm, Dynamic structure factor: An introduction, Z. Naturforsch. A 48, 233 (1993).

[19] S. Galambosi, L. Wirtz, J. A. Soininen, J. Serrano, A. Marini, K. Watanabe, T. Taniguchi, S. Huotari, A. Rubio, and K. Hämäläinen, Anisotropic excitonic effects in the energy loss function of hexagonal boron nitride, Phys. Rev. B 83, 081413 (2011).

[20] G. Fugallo, M. Aramini, J. Koskelo, K. Watanabe, T. Taniguchi, M. Hakala, S. Huotari, M. Gatti, and F. Sottile, Exciton energymomentum map of hexagonal boron nitride, Phys. Rev. B 92, 165122 (2015).

[21] See Supplemental Material at http://link.aps.org/supplemental/ 10.1103/PhysRevB.96.115304 for TEM images of the sample, $K$-edge data on nitrogen and further details on the geometry of the $\omega-\mathbf{q}$ acquisition method and on the theoretical calculations.

[22] M. Haider, S. Uhlemann, E. Schwan, H. Rose, and B. Kabius, Electron microscopy image enhanced, Nature (London) 392, 768 (1998).

[23] O. L. Krivanek, N. Dellby, and A. R. Lupini, Towards sub- $\AA$ electron beams, Ultramicroscopy 78, 1 (1999).

[24] M. Matijevic, H. Stegmann, E. Essers, and G. Benner, Recent Results of an In-Column Energy Filter TEM with Monochromator, Microsc. Microanal. 13, 1160 (2007).

[25] D. A. Muller, L. F. Kourkoutis, M. Murfitt, J. H. Song, H. Y. Hwang, J. Silcox, N. Dellby, and O. L. Krivanek, Atomic-scale chemical imaging of composition and bonding by aberrationcorrected microscopy, Science 319, 1073 (2008).

[26] K. Suenaga and M. Koshino, Atom-by-atom spectroscopy at graphene edge, Nature (London) 468, 1088 (2010).

[27] C. Jeanguillaume and C. Colliex, Spectrum-image: The next step in EELS digital acquisition and processing, Ultramicroscopy 28, 252 (1989). 
[28] L. Reimer, I. Fromm, and R. Rennekamp, Operation modes of electron spectroscopic imaging and electron energyloss spectroscopy in a transmission electron microscope, Ultramicroscopy 24, 339 (1988).

[29] L. Reimer and R. Rennekamp, Imaging and recording of multiple scattering effects by angular-resolved electron energy-loss spectroscopy, Ultramicroscopy 28, 258 (1989).

[30] P. Wachsmuth, R. Hambach, M. K. Kinyanjui, M. Guzzo, G. Benner, and U. Kaiser, High-energy collective electronic excitations in free-standing single-layer graphene, Phys. Rev. B 88, 075433 (2013).

[31] T. Taniguchi and K. Watanabe, Synthesis of high-purity boron nitride single crystals under high pressure by using Ba-BN solvent, J. Cryst. Growth 303, 525 (2007).

[32] R. D. Leapman, P. L. Fejes, and J. Silcox, Orientation dependence of core edges from anisotropic materials determined by inelastic scattering of fast electrons, Phys. Rev. B 28, 2361 (1983).

[33] S. Saito, K. Higeta, and T. Ichinokawa, Intensity analysis of boron and nitrogen $K$-edge spectra for hexagonal boron nitride by EELS, J. Microsc. (Oxford, UK) 142, 141 (1986).

[34] N. Watanabe, H. Hayashi, Y. Udagawa, K. Takeshita, and H. Kawata, Anisotropy of hexagonal boron nitride core absorption spectra by x-ray raman spectroscopy, Appl. Phys. Lett. 69, 1370 (1996).

[35] R. Franke, S. Bender, J. Hormes, A. A. Pavlychev, and N. G. Fominych, A quasi-atomic treatment of chemical and structural effects on K-shell excitations in hexagonal and cubic BN crystals, Chem. Phys. 216, 243 (1997).

[36] M. Jaouen, G. Hug, B. Ravel, A. L. Ankudinov, and J. J. Rehr, Polarisation effects in hexagonal boron nitride near-edge structure: A real-space multiple scattering approach, Europhys. Lett. 49, 343 (2000).

[37] R. Arenal, M. Kociak, and N. J. Zaluzec, High-angularresolution electron energy loss spectroscopy of hexagonal boron nitride, Appl. Phys. Lett. 90, (2007).

[38] Y. Feng, J. A. Soininen, A. L. Ankudinov, J. O. Cross, G. T. Seidler, A. T. Macrander, J. J. Rehr, and E. L. Shirley, Exciton spectroscopy of hexagonal boron nitride using nonresonant x-ray raman scattering, Phys. Rev. B 77, 165202 (2008).

[39] N. L. McDougall, R. J. Nicholls, J. G. Partridge, and D. G. McCulloch, The near edge structure of hexagonal boron nitride, Microsc. Microanal. 20, 1053 (2014).

[40] J. Wang, Z. Wang, H. Cho, M. J. Kim, T. K. Sham, and X. Sun, Layer speciation and electronic structure investigation of freestanding hexagonal boron nitride nanosheets, Nanoscale 7, 1718 (2015)

[41] G. Strinati, Application of the Green's functions method to the study of the optical properties of semiconductors, Riv. Nuovo Cimento Soc. Ital. Fis. 11, 1 (1988).
[42] D. Pacilé, M. Papagno, T. Skála, V. Matolín, T. Sainsbury, T. Ikuno, D. Okawa, A. Zettl, and K. C. Prince, Excitons at the B $K$ edge of boron nitride nanotubes probed by $\mathrm{X}$-ray absorption spectroscopy, J. Phys. Condens. Matter 22, 295301 (2010).

[43] C. Tarrio and S. E. Schnatterly, Interband transitions, plasmons, and dispersion in hexagonal boron nitride, Phys. Rev. B 40, 7852 (1989).

[44] P. Moreau and M. C. Cheynet, Improved comparison of low energy loss spectra with band structure calculations: The example of BN filaments, Ultramicroscopy 94, 293 (2003).

[45] A. G. Marinopoulos, L. Wirtz, A. Marini, V. Olevano, A. Rubio, and L. Reining, Optical absorption and electron energy loss spectra of carbon and boron nitride nanotubes: A first-principles approach, Appl. Phys. A 78, 1157 (2004).

[46] R. J. Nicholls, J. M. Perkins, V. Nicolosi, D. W. McComb, P. D. Nellist, and J. R. Yates, Low-loss EELS of 2D boron nitride, J. Phys. Conf. Ser. 371, 012060 (2012).

[47] C. T. Pan, R. R. Nair, U. Bangert, Q. Ramasse, R. Jalil, R. Zan, C. R. Seabourne, and A. J. Scott, Nanoscale electron diffraction and plasmon spectroscopy of single- and few-layer boron nitride, Phys. Rev. B 85, 045440 (2012).

[48] F. J. Nelson, J.-C.s Idrobo, J. D. Fite, Z. L. Mišković, S. J. Pennycook, S. T. Pantelides, J. U. Lee, and A. C. Diebold, Electronic excitations in graphene in the $1-50 \mathrm{ev}$ range: The $\pi$ and $\pi+\sigma$ peaks are not plasmons, Nano Lett. 14, 3827 (2014).

[49] D. Novko, V. Despoja, and M. Šunjić, Changing character of electronic transitions in graphene: From single-particle excitations to plasmons, Phys. Rev. B 91, 195407 (2015).

[50] S. C. Liou, C.-S. Shie, C. H. Chen, R. Breitwieser, W. W. Pai, G. Y. Guo, and M.-W. Chu, $\pi$-plasmon dispersion in freestanding graphene by momentum-resolved electron energy-loss spectroscopy, Phys. Rev. B 91, 045418 (2015).

[51] V. U. Nazarov, Electronic excitations in quasi-2D crystals: What theoretical quantities are relevant to experiment? New J. Phys. 17, 073018 (2015)

[52] H.-C. Weissker, J. Serrano, S. Huotari, E. Luppi, M. Cazzaniga, F. Bruneval, F. Sottile, G. Monaco, V. Olevano, and L. Reining, Dynamic structure factor and dielectric function of silicon for finite momentum transfer: Inelastic x-ray scattering experiments and $a b$ initio calculations, Phys. Rev. B 81, 085104 (2010).

[53] S. Latini, T. Olsen, and K. S. Thygesen, Excitons in van der Waals heterostructures: The important role of dielectric screening, Phys. Rev. B 92, 245123 (2015).

[54] G. Cappellini, G. Satta, M. Palummo, and G. Onida, Optical properties of $\mathrm{BN}$ in cubic and layered hexagonal phases, Phys. Rev. B 64, 035104 (2001).

[55] G. Y. Guo and J. C. Lin, Systematic ab initio study of the optical properties of BN nanotubes, Phys. Rev. B 71, 165402 (2005).

[56] J. J. Mortensen, L. B. Hansen, and K. W. Jacobsen, Real-space grid implementation of the projector augmented wave method, Phys. Rev. B 71, 035109 (2005).

[57] J. P. Perdew, K. Burke, and M. Ernzerhof, Generalized Gradient Approximation Made Simple, Phys. Rev. Lett. 77, 3865 (1996). 